\title{
Chemsex: ¿estamos preparados?
}

\section{Chemsex: are we prepared?}

\author{
David Redondo Domínguez*, Luis Picazo*, María Luisa Docavo Barrenechea-Moxo*, \\ Juan González del Castillo*,**. \\ * Servicio de Urgencias, Hospital Clínico San Carlos, Madrid, España. ** Instituto de Investigación Sanitaria \\ del Hospital Clínico San Carlos, Madrid, España.
}

$\mathrm{E}$ stimado Sr Editor,

Hemos leído con gran interés el artículo publicado por Dolengevich-Segal (Dolengevich-Segal et al., 2017) donde se hace una interesante revisión sobre un fenómeno emergente como es el chemsex, y las diferentes drogas que se utilizan en este tipo de prácticas.

Nos gustaría poner de manifiesto la importancia y utilidad de la publicación, por el progresivo incremento en la prevalencia del consumo de drogas en el contexto de la actividad sexual en hombres que mantienen sexo con hombres (HSH) en Europa Occidental (Fernández-Dávila et al,. 2016). Esto conduce a la necesidad de poner en marcha estudios epidemiológicos que evalúen el fenómeno, pero no solo desde el punto de vista de salud mental, sino también desde el campo de las enfermedades infecciosas y de la toxicología. No debemos olvidar que este tipo de prácticas suponen un incremento en el riesgo de infección por enfermedades de transmisión sexual y los problemas desde el punto de vista toxicológico al que pueden dar lugar el consumo de drogas. Ambos constituyen motivos de consulta en los servicios de urgencias (SU), donde debería incrementarse la formación necesaria para abordar los problemas derivados del chemsex.

La medicación es la segunda causa de las visitas al SU (Bilbao Gómez-Martino et al., 2017). Además del impacto en la seguridad de los pacientes, los efectos adversos conllevan una mayor utilización de los recursos sanitarios
(Bilbao Gómez-Martino et al., 2017). Las intoxicaciones agudas son un motivo frecuente de consulta en estos servicios, representando un 0,5-1\% del total de visitas (Supervía Caparrós et al., 2017). Por estos motivos, los SU deberían proponer programas de investigación e intervención sobre esta problemática, utilizando sus propias redes de investigación y siempre con la colaboración y la sintonía de los expertos en toxicología (Burbano Santos et al., 2017; González Del Castillo et al., 2017).

El consumo de este tipo de sustancias con usos recreativos (mefedrona, GHB y metanfetamina), frecuentes en las sesiones de chemsex, constituye un riesgo para la salud, incrementado por la inexistencia de un antídoto específico para ninguna de ellas (Coll et al., 2016). Además, al ser drogas que se han extendido por este colectivo recientemente, han sido poco estudiadas y se sabe muy poco acerca de su toxicidad y problemas derivados de su consumo a largo plazo. La metanfetamina por vía intranasal puede ser un factor de riesgo para la transmisión del virus de la hepatitis C tras compartir el material (turulo), sin olvidar que la vía de administración más peligrosa es la inyectada (Folch et al., 2015). Por otra parte, los inhibidores de la proteasa pueden aumentar los niveles de GHB, y las metanfetaminas usan la misma ruta metabólica hepática que éstos, por lo que existen altas posibilidades de que hayan interacciones graves entre ellas (Hales et al., 2000). Se han descrito muertes en pacientes con VIH que toman metanfetamina durante el tratamiento con ritonavir (Hales et al., 2000). El

Recibido: Noviembre 2017; Aceptado: Noviembre 2017

Enviar correspondencia a:

Juan González del Castillo. Servicio de Urgencias. Hospital Clínico San Carlos. Calle Profesor Martín-Lagos s/n, 28040 Madrid. Tel: (34) 9133037 50. Fax: (34) 9133035 69. Email: jgonzalezcast@gmail.com 
número de casos descritos es pequeño, pero probablemente el riesgo es significativo para personas en tratamiento con ritonavir y cobicistat.

Por otra parte, y considerando el incremento de las primoinfecciones de VIH en HSH, algunos profesionales sanitarios plantean la profilaxis pre-exposición (PrEP), una intervención de tipo biomédico, como manera rentable de reducir la transmisión del VIH entre los hombres de este grupo de alto riesgo y más barata que la profilaxis post-exposición (PEP) y la toma de antirretrovirales de por vida. La PrEp, realizada habitualmente con Truvada, introduce un nuevo fármaco en el cóctel y podría tener un efecto sumatorio de toxicidad o de pérdida de eficacia con el policonsumo de las drogas utilizadas en las sesiones de chemsex debido a las potenciales interacciones (Uglietti et al., 2012).

En base a lo anteriormente expuesto, creemos necesario realizar estudios de investigación, aumentar la formación de los médicos de los SU sobre estos aspectos e incrementar las campañas de información a la población en relación a los efectos de todas estas sustancias, de aparición reciente en comparación a otras drogas más conocidas, así como de los riesgos que asocian los contextos en los que se utilizan. Ante la tendencia creciente de este tipo de prácticas sexuales, estas campañas podrían dirigirse no solo a la población diana, sino además involucrar a locales de ocio y personal sanitario para promover la terapia educacional desde atención primaria hasta los SU.

\section{Conflictos de interés}

Los autores de este artículo declaramos que no existe conflicto de intereses.

\section{Referencias}

Dolengevich-Segal, H., Rodriguez-Salgado, B., Bellesteros-Lopez, J. y Molina-Prado, R. (2017). Chemsex. An emergent phenomenon. Adicciones, 29, 207-209. doi:10.20882/adicciones.894.

Fernández-Dávila, P. (2016). "Sesión de sexo, morbo y vicio": una aproximación holística para entender la aparición del fenómeno Chemsex entre hombres gais, bisexuales y otros hombres que tienen sexo con hombres en España. Revista Multidisciplinar del Sida, 4, 41-65.

Bilbao Gómez-Martino, C., Nieto Sánchez, A., Fernández Pérez, C, Borrego Hernando, MI. y Martín-Sánchez, FJ. (2017). Perfil de riesgo y análisis comparativo de los errores de conciliación de medicamentos según el médico prescriptor y la herramienta de prescripción. Emergencias, 29, 384-390.

Supervia Caparros, A., Pallas Villaronga, O., Clemente Rodriguez, C., Aranda Cardenas, M. D., Pi-Figueras Valls, M. y Cirera Lorenzo, I. (2017). Emergencias, 29, 335-338.
Burbano Santos, P., Fernandez-Guerrero, I. M., Martin-Sanchez, F. J., Burillo, G. y Miro, O. (2017). Emergencias, 29, 320-326.

Gonzalez Del Castillo, J. y Martin-Sanchez, F. J. (2017). Microorganismos resistentes en urgencias: ¿cómo afrontar el reto? Emergencias, 29, 303-305.

Coll J, F. C. (2016). Drogas recreativas y sexo en hombres que tienen sexo con hombres: chemsex. Riesgos, problemas de salud asociados a su consumo, factores emocionales y estrategias de intervención. Enfermedades Emergentes, 15, 77-84.

Folch, C., Fernandez-Davila, P., Ferrer, L., Soriano, R., Diez, M. y Casabona, J. (2015). Medicina Clínica, 145, 102-107. doi:10.1016/j.medcli.2014.04.030.

Hales, G., Roth, N. y Smith, D. (2000). Possible fatal interaction between protease inhibitors and methamphetamine. Antiviral Therapy, 5, 19.

Uglietti, A., Zanaboni, D., Gnarini, M. y Maserati, R. (2012). Emtricitabine/tenofovir in the treatment of HIV infection: current PK/PD evaluation. Expert Opinion on Drug Metabolism Eे Toxicology, 8, 1305-1314. doi:10.1517/1742 5255.2012 .714367$. 